\title{
Synthesis and Characterization of Bismuth Based Novel Inorganic Ion Exchange Materials and Their Analytical Applications.
}

\author{
Siji $\mathrm{S}^{1}$, Janardanan $\mathrm{C}^{2}$ \\ ${ }_{1 \& 2}$ (Post Graduate and Research Department of Chemistry, \\ Sree Narayana College, Kannur-670 007, Kerala, India)
}

\begin{abstract}
Two new inorganic ion exchange materials Bismuth(III) antimonotungstate(BiSbW) and Bi(III) iodotungstate(BiIW), salts of heteropoly acids were compared to evaluate their performance as ion exchangers and to explore their efficacy as dye adsorbents. Amorphous BiSbW and BiIW have been synthesized by coprecipitation methods by varying the mixing ratio of the reagents. The most stable samples of BiIW and BiSbW have been characterized for spectral analysis (FTIR), elemental analysis (SEM-EDS), thermal analysis (TGA and DTA) and X-ray diffraction studies. Their ion exchange capacities for $\mathrm{Na}^{+}$ions were found to be 0.85 meqg $^{-1}(B i S b W)$ and 2.25 meqg $^{-1}(B i I W)$. The effect on the exchange capacity of drying the exchanger at different temperatures has been studied. The distribution coefficient values shows that these two samples are very selective for $\mathrm{Pb}^{2+}$ ions. Hence their analytical importance has been established by quantitative separation of $\mathrm{Pb}^{2+}$ from other metal ions and quantitative removal of $\mathrm{Pb}^{2+}$ ions from textile industry. The adsorption results also reflected the vital influence of the new materials in controlling the hazardous organic pollutants by adsorption of various dyes from waste water.
\end{abstract}

Keywords: cation exchangers, distribution coefficient, dye adsorbents, heteropoly acids, ion exchange capacity etc

\section{INTRODUCTION}

The contamination of water wealth due to the unsystematic dumping of heavy metals and hazardous organic pollutants has been causing international anxiety for the last few decades. Diverse management techniques for wastewater loaded with heavy metals have been developed in recent years both to reduce the amount of wastewater created and to improve the quality of the treated effluents. Nowadays ion exchangers have received prominent position in water processing and in the chemical engineering. Ion exchangers as a separation process is comparatively too simple and energy -efficient compared to other established waste treatment techniques. Inorganic ion exchangers appeared as potential advanced materials to this challenging task exceptionally well, as they have effective properties such as good ion exchange characteristics, thermal stability and chemical resistivity [1-3]. Application of selective inorganic ion exchangers is a known way for recovery of heavy metals as they exhibit high affinities for specific ions [4-5].

The present work is concerned with Bismuth based three-component ion exchangers. By a review of the literature it is obvious that little research works have been done in the field of bismuth based materials as ion exchangers. Bismuth tungstate[6], bismuth nitrate[7], bismuth tellurate[8], bismuth silicate[9], bismuth(III) iodophosphate[10], bismuth(III) tungstosilicate[11] and bismuth(III) tungstomolybdate[12] etc have been studied for their synthesis, ion-exchange behaviour and analytical applications. In this manuscript, we describe the synthesis and ion exchange properties of two new inorganic ion exchange materials, Bismuth(III) antimonotungstate and Bismuth(III) iodotungstate. As per the literature no attempt has been reported on bismuth(III) antimonotungstate and bismuth(III) iodotungstate as ion exchangers. It was found that these exchanger exhibits an extremely high affinity for lead ion in the presence of an excess of other competitive species and, hence, could be regarded as promising materials for the detection of trace amount of lead ion in contaminated water and for the removal and recovery of the same from waste water. Moreover their efficacy intended for adsorption of different dyes from dye effluents was also examined and studied using UV-Vis Visible Diffuse Reflectance spectrometer.

\section{Reagents and chemicals}

\section{EXPERIMENTAL}

Bismuth nitrate, sodium tungstate, potassium pyroantimonate and potassium iodate were obtained from E.Merck (India). All other chemicals and reagents used were of analytical grade. 


\section{Instrumentation}

pH measurements were performed using an ELICO LI613 pH meter. Spectrophotometry was done on a UV- Visible Spectrophotometer model JASCO V660 with diffuse reflectance accessory (integrated sphere). IR studies were made using an FTIR spectrometer model Thermo Nicolet Avtar370and thermogram was run on Perkin Elmer Diamond TG/DTA Analyzer. X-ray diffractometer BrukerAXS D8 Advance for X-ray diffraction studies and an electric temperature controlled shaker was used for shaking. Chemical composition was determined using EDS. A glass column was used for column operations

\section{Synthesis of the exchanger}

Different samples of BiSbW and BiIW were prepared by adding 0.1M Bismuth nitrate solution to a mixture of $0.05 \mathrm{M}$ sodium tungstate solution and $0.05 \mathrm{M}$ potassium pyroantimonate solution and to a mixture of $0.05 \mathrm{M}$ sodium tungstate solution and $0.05 \mathrm{M}$ potassium iodate solution respectively in different volume ratios with intermittent shaking of the mixture and keeping the $\mathrm{pH}$ at 1.0 as given in table 1 and 2.The precipitates were filtered, washed with deionized water and dried. The exchangers were then converted in to the $\mathrm{H}^{+}$form by treating with $1 \mathrm{M}$ nitric acid for $24 \mathrm{hrs}$ with occasional shaking and intermittent changing of acid. Then the samples were washed with deionized water to remove the excess acid, dried and sieved to obtain particles of 60100 mesh.

\subsection{Ion exchange capacity}

The IEC of the material was determined by the column process [13]; $1.0 \mathrm{~g}$ of the exchanger $\left(\mathrm{H}^{+}\right.$form) was packed in a glass column of $1.1 \mathrm{~cm}$ diameter. The $\mathrm{H}^{+}$ions were eluted by adding $100 \mathrm{ml}$ of $1 \mathrm{M}$ sodium chloride solution. The effluent was collected and titrated against standard sodium hydroxide solution. The exchange capacity in meq/g was calculated using the formula:

$$
\text { IEC }=\frac{a v}{w}
$$

Where ' $a$ ' is the molarity, ' $v$ ' is the volume of alkali used during titration and ' $w$ ' is the weight of the exchanger taken.

\section{2. pH titration}

Topp and Pepper method [14] was used for $\mathrm{pH}$ titration using $\mathrm{NaOH} / \mathrm{NaCl}, \mathrm{KOH} / \mathrm{KCl}$ systems. $0.5 \mathrm{~g}$ of exchanger was equilibrated with varying amounts of metal chloride and metal hydroxide solutions. After equilibrium the $\mathrm{pH}$ of each solution was measured and plotted against milliequivalents of $\mathrm{OH}^{-}$ions.

\subsection{Chemical stability}

The chemical stability of the exchanger was assessed in mineral acid like $\mathrm{HCl}, \mathrm{HNO}_{3}$, and $\mathrm{H}_{2} \mathrm{SO}_{4}$, bases like $\mathrm{NaOH}$ and $\mathrm{KOH}$ and organic solvents like acetic acid, acetone, ethanol and diethyl ether. For this $500 \mathrm{mg}$ of the exchanger was kept separately in $50 \mathrm{ml}$ of different solvents at room temperature for 24 hours. The change in colour and weight were noted.

\subsection{Effect of temperature}

The effect of temperature on ion exchange capacity was studied by heating several $1 \mathrm{~g}$ sample of the exchanger at different temperatures for three hours in an air oven and $\mathrm{Na}^{+}$ion exchange capacity in meq $\mathrm{g}^{-1}$ was determined by the column method after cooling them to room temperature.

\subsection{Distribution studies $\left(\mathbf{K}_{\mathbf{d}}\right)$}

Distribution studies were carried out for various metal ions in demineralised water by batch process. In this process $100 \mathrm{mg}$ of the dry exchanger in $\mathrm{H}^{+}$form were equilibrated with $20 \mathrm{ml}$ of different metal ion solutions and kept for 24 hours at room temperature. The determination before and after equilibrium were carried out volumetrically using EDTA as the titrant. The $\mathrm{K}_{\mathrm{d}}$ values as depicted in Fig.7, were obtained by the formula:

$$
\mathrm{K}_{\mathrm{d}}=\frac{I-F}{F} \times \frac{v}{w}
$$

Where 'I' is the initial volume of EDTA used, ' $F$ ' is the final volume of EDTA used; ' $V$ ' is the volume of the metal ion solution and ' $\mathrm{W}$ ' is the weight of the exchanger.

\subsection{Binary separations}

Separations of some metal ions of analytical utility were achieved on the column of the exchangers by knowing the effect of electrolyte concentration on distribution coefficients. This was studied by equilibrating known amount of exchanger with metal ion solution in electrolytes of varying concentrations $(0.1 \mathrm{M}, 0.01 \mathrm{M}$, 
$0.001 \mathrm{M} \mathrm{NH}_{4} \mathrm{NO}_{3}(\mathrm{AN})$ and $\left.\mathrm{HNO}_{3}(\mathrm{NA})\right)$. The column $(30 \mathrm{~cm} \times 0.69 \mathrm{~cm}$ internal diameter) on which the separations were to be carried out was filled uniformly with the exchanger $(5 \mathrm{~g})$. First of all distilled water was added to pack the granules so that no air bubbles get stuck. Then the mixture of the metal ion solutions of concentration about $0.005 \mathrm{M}(10 \mathrm{ml})$ was slowly added. The process was repeated for maximum sorption. The exchanged metal ions were eluted using suitable eluent. The rate of flow in all separations was $0.2 \mathrm{ml}$ per minute. The concentration of metal ion in the solution was determined by collecting known volume of effluent at regular intervals. Binary separations were carried out on the column.

\subsection{Recovery of lead from textile wastewater}

Samples of waste water were collected from various textile industries. The waste water samples were filtered first to remove suspended particle and then neutralised. The solution was decolourised using charcoal. The samples thus obtained were used to separate and recover the heavy metal present in it. The ion exchange materials having greater selectivity towards lead metal ions were packed in a column, $100 \mathrm{ml}$ of the sample was passed through the column and repeated 3-4 times using effluent collected at the bottom. This was for the maximum uptake of cations. The flow rate was maintained at $0.2 \mathrm{ml}$ per minute. Varying concentrations of $\mathrm{NH}_{4} \mathrm{NO}_{3}$ and $\mathrm{HNO}_{3}$ as eluents were used to elute the metal ions completely from the cation exchanger column and then determined titrimetrically with standard EDTA solution.

\subsection{Adsorption of dyes:}

Aqueous solution of methyl orange (MO) and methylene blue (MB) (20 ml, 20ppm) were equilibrated separately with $300 \mathrm{mg}$ of exchanger for $24 \mathrm{~h}$. The sample solutions were then filtered and centrifuged to remove the adsorbent prior to analysis. Absorbance was recorded before and after equilibration with a UV-Visible Diffuse Reflectance spectrometer at the absorption maximum of each dye (MO (463nm) and MB (665nm)).

\section{BiSbW and BiIW as inorganic cation exchange materials. \\ III. Results And Discussion}

BiSbW and BiIW appear to be potential ion exchangers having good ion exchange capacity (IEC). Table 1\&2 describes the preparation and properties of the materials. For BiSbW, sample BiSbW-6 and for BiIW, sample BiIW-4 were chosen for detailed studies due to their higher IEC and chemical stability.

The energy dispersive spectrometry (EDS) was used to identify the elements exist in the prepared sample by taking a selective portion of SEM image in the form of peaks of spectrum. The composition of the BiSbW and BiIW was found as Bi: Sb: W; 1:1.4:1.01 and Bi: I: W; 1.8: 1.4: 1

The $\mathrm{pH}$ titration curve (Fig. 1) shows that the ion exchange materials release $\mathrm{H}^{+}$ions easily on addition of $\mathrm{NaCl}$ and $\mathrm{KCl}$ solution to the system in neutral medium, which is indicated by a $\mathrm{pH}$ value $\sim 3.5$ of the solution. As the volume of $\mathrm{NaOH}$ added to the system is increased, more $\mathrm{OH}^{-}$ions are consumed suggesting in the increase of the rate of ion exchange in basic medium due to the removal of $\mathrm{H}^{+}$ions from the external solution. Further, the titration curve showed a bifunctional behavior of the exchangers. The IEC calculated from the titration curve is in close agreement to those obtained by the column method.

The effect of heating on IEC of BiSbW has been compared with that of similarly treated BiIW. It is apparent from Fig. 2 that even though the BiIW has the highest IEC, the retention of IEC was higher for BiSbW as the samples were dried at $50{ }^{\circ} \mathrm{C}$ and this trend follows up to $300{ }^{\circ} \mathrm{C}$. This indicates the thermal stability of BiSbW as contrast to BiIW.

The materials were found to be stable in $1.0 \mathrm{M} \mathrm{HCl}, \mathrm{HNO}_{3}, \mathrm{H}_{2} \mathrm{SO}_{4}$ and in $0.1 \mathrm{M}$ solutions of $\mathrm{KOH}$ and $\mathrm{NaOH}$. The materials were also reasonably stable in organic solvents like methanol, ethanol, acetone, carbon tetrachloride, diethyl ether etc.

The TGA of BiSbW (Fig. 3a) indicates the presence of hydrated water which is lost up to $95^{\circ} \mathrm{C}$. A gradual weight loss observed up to $370^{\circ} \mathrm{c}$ is due to the condensation of exchangeable hydroxyl groups which is the usual behavior of inorganic ion exchangers $[15,16]$. After that no sharp change in weight indicates the stability of the compound. The total weight loss up to $900^{\circ} \mathrm{C}$ is only $13 \%$.

The thermogram of BiIW (Fig. 3b) reveals some remarkable information. The external water molecules are lost up to $180{ }^{\circ} \mathrm{C}$ as indicated by the first weight loss of $7.74 \%$. Further, a gradual loss of weight of Ion exchanger was attributed to the condensation of $\mathrm{IO}_{3}{ }^{-}$group in to $\mathrm{I}_{2} \mathrm{O}_{5}[17,18]$. The decrease in IEC on increasing temperature is due to the conversion of $\mathrm{IO}_{3}{ }^{-}$into $\mathrm{I}_{2} \mathrm{O}_{5}$. Beyond $500{ }^{\circ} \mathrm{C}$, the weight becomes constant owing to the formation of metal oxide.

To assist the advance characterization of the materials, their FTIR studies (Fig. 4) were performed at room temperature. The strong and broad band in the region $3600-3000 \mathrm{~cm}^{-1}$ present in two materials may be assigned to interstitial water molecule and $\mathrm{OH}^{-}$group $[19,20]$. A weak band in the region $2360 \mathrm{~cm}^{-1}$ common to both spectrums was due to deformation vibration of the coordinated water molecule. Another strong and sharp peak with maximum at $1619 \mathrm{~cm}^{-1}$ may be due to $\mathrm{H}-\mathrm{O}-\mathrm{H}$ bending and band at $1383 \mathrm{~cm}^{-1}$ was due to deformation 
vibrations of metal hydroxyl groups and interstitial water. The spectrum also shows strong band in the region $715 \mathrm{~cm}^{-1}$ indicating the presence of tungstate and other bands in the region $800-420 \mathrm{~cm}^{-1}$ was attributed to metal oxygen vibrations [21-23].

$\mathrm{X}$-ray diffraction pattern of $\mathrm{BiSbW}$ and $\mathrm{BiIW}$ in $\mathrm{H}^{+}$form reveals the fact that the exchangers are amorphous (Fig. 5).

SEM images of BiSbW and BiIW (Fig. 6) reveal that the particles having an irregular shape and there is no sign of crystalline structure and these observations strongly support the amorphous nature of the materials.

Distribution studies of metal ions (Fig .7) were carried out individually with the samples. Sorption of ions was found to be varied in different samples of exchanger. The distribution studies showed that the materials were found to possess exceptional high $K_{d}$ values for $\mathrm{Pb}^{2+}$ and hence considered to be highly selective for $\mathrm{Pb}^{2+}$. $\mathrm{Pb}$ (II) has been considered as a major polluting material in water. The separation capacity of these materials has been demonstrated by achieving some important binary separations such as $\mathrm{Pb}$ (II)- $\mathrm{Hg}$ (II) and $\mathrm{Pb}$ (II)- $\mathrm{Ni}(\mathrm{II})$ effectively.

In order to select suitable eluents for each metal ion, distribution studies were carried out in different electrolytes. Varying molar concentrations of ammonium nitrate (AN) and nitric acid (NA) were used for this study. No significant sorption for $\mathrm{Hg}$ (II) and $\mathrm{Ni}(\mathrm{II})$ ions was noted in these electrolytes. Sorption of $\mathrm{Pb}$ (II) ions was found to be varied in different electrolyte solutions (Fig. 8). Table $3 \& 4$ summarizes the salient features of these separations. The separations are rather sharp and recovery is quantitative and reproducible.

Distribution coefficient $\left(\mathrm{K}_{\mathrm{d}}\right)$ values were used as a measure of the selectivity of the materials for lead metal ions. The selectivity determines how competently the element of concern is separated from other elements. In order to reveal the practical usefulness of the material, quantitative separations of $\mathrm{Pb}$ (II) from textile industry effluents have been achieved using BiIW and BiSbW (table 5).

\section{BiSbW and BiIW as efficient dye adsorbents}

Again the analytical applications of the materials were studied by controlling the hazardous organic pollutants in waste water by adsorption of Methylene blue and methyl orange on these ion exchange surfaces. UV-Visible absorption spectrum of methyl orange and methylene blue before and after equilibration with the sorbents are shown in Fig. 9. The drastic change in the intensity of each peak at absorption maxima reveals the effectiveness of the adsorbents for removing these dyes from aqueous solution. Greater than $95 \%$ of Methylene blue and $80 \%$ of Methyl orange dyes were removed within 24 hours under stationary conditions. Since BiIW and BiSbW hold structural hydroxyl group, the dyes could be bond to the sorbents with hydrogen bonds or weak Van der Waal's forces. The high adsorption for methylene blue could be due to the positive charge on the hetero atom. Even small changes in the dye structure can significantly influence its adsorption capacity. In the spectrum of MO, the absorption maxima was shifted to higher wavelength region due to the change in $\mathrm{pH}$ of dye solution by the addition of weak acidic cation exchanger. The efficiency of BiIW and BiSbW towards removal of methyl orange and methylene blue from aqueous solution indicates their promising use in waste water containing dyes. Table 6 illustrates the experimental and calculated data for the removal of MB and MO from synthetic wastewater.

\section{Figures AND TABLES}

Table-1 Conditions of synthesis and properties of different samples of Bismuth(III) antimonotungstate

\begin{tabular}{|c|c|c|c|c|c|c|c|}
\hline \multirow{2}{*}{ sample } & \multicolumn{3}{|c|}{ Molar conc.(M) } & \multirow{2}{*}{$\begin{array}{c}\text { Volume } \\
\text { mixing } \\
\text { ratio }\end{array}$} & \multirow{2}{*}{$\mathbf{p H}$} & \multirow{2}{*}{ Appearance } & \multirow{2}{*}{ I.E.C(meq/g) } \\
\hline & $\mathrm{Bi}$ & $\mathrm{Sb}$ & $\mathrm{W}$ & & & & \\
\hline BiSbW1 & 0.05 & 0.05 & 0.05 & $1: 1: 1$ & 1 & \multirow{6}{*}{ All forms white solids } & 0.57 \\
\hline BiSbW2 & 0.05 & 0.05 & 0.05 & $1: 2: 1$ & 1 & & 0.55 \\
\hline BiSbW3 & 0.05 & 0.05 & 0.05 & $1: 1: 2$ & 1 & & 0.77 \\
\hline BiSbW4 & 0.05 & 0.05 & 0.05 & $1: 1: 3$ & 1 & & 0.70 \\
\hline BiSbW5 & 0.05 & 0.05 & 0.05 & $1: 3: 1$ & 1 & & 0.82 \\
\hline BiSbW6 & 0.05 & 0.05 & 0.05 & $1: 2: 3$ & 1 & & 0.85 \\
\hline
\end{tabular}


Table-2 Conditions of synthesis and properties of different samples of Bismuth(III) iodotungstate

\begin{tabular}{cccccccc}
\hline sample & \multicolumn{2}{c}{ Molar conc.(M) } & $\begin{array}{c}\text { Volume } \\
\text { mixing } \\
\text { ratio }\end{array}$ & pH & Appearance & I.E.C(meq/g) \\
\hline BiIW1 & 0.05 & 0.05 & 0.05 & $1: 1: 1$ & 1 & & \\
BiIW2 & 0.05 & 0.05 & 0.05 & $1: 2: 1$ & 1 & All forms white & 1.75 \\
BiIW3 & 0.05 & 0.05 & 0.05 & $1: 1: 2$ & 1 & solids & 0.85 \\
BiIW4 & 0.05 & 0.05 & 0.05 & $1: 1: 3$ & 1 & & 2.25 \\
BiIW5 & 0.05 & 0.05 & 0.05 & $1: 3: 1$ & 1 & & 0.98 \\
BiIW6 & 0.05 & 0.05 & 0.05 & $1: 2: 3$ & 1 & & 1.25 \\
\hline
\end{tabular}

Table-3 Binary separation of metal ions on Bismuth iodotungstate

\begin{tabular}{c|c|c|c|c|c}
\hline $\begin{array}{c}\text { Separations } \\
\text { achieved }\end{array}$ & $\begin{array}{c}\text { Separation } \\
\text { factor }\end{array}$ & Eluent & \multicolumn{2}{|c|}{ Metal ion(mg) } & \% efficiency \\
\cline { 4 - 5 } $\mathrm{Hg}(\mathrm{II})$ & 26.32 & $0.01 \mathrm{M} \mathrm{AN}$ & 5.01 & 4.87 & 97.21 \\
$\mathrm{~Pb}(\mathrm{II})$ & & $1 \mathrm{M} \mathrm{NA}+0.1 \mathrm{M} \mathrm{AN}$ & 4.04 & 3.91 & 96.78 \\
$\mathrm{Ni}(\mathrm{II})$ & 75.91 & $0.01 \mathrm{M} \mathrm{AN}$ & 1.39 & 1.34 & 96.40 \\
$\mathrm{~Pb}(\mathrm{II})$ & & 1M NA+0.1M AN & 4.04 & 3.98 & 98.51 \\
& & & & & \\
\hline
\end{tabular}

Table-4 Binary separation of metal ions on Bismuth antimonotungstate

\begin{tabular}{c|c|c|c|c|c}
\hline $\begin{array}{c}\text { Separations } \\
\text { achieved }\end{array}$ & $\begin{array}{c}\text { Separation } \\
\text { factor }\end{array}$ & Eluent & \multicolumn{2}{|c|}{ Metal ion(mg) } & \% efficiency \\
\cline { 4 - 5 } & 141.89 & $0.01 \mathrm{M} \mathrm{AN}$ & Loaded & Eluted & \\
\hline $\mathrm{Hg}(\mathrm{II})$ & & $1 \mathrm{M} \mathrm{NA}+0.1 \mathrm{M} \mathrm{AN}$ & 4.04 & 4.42 & 88.23 \\
$\mathrm{~Pb}(\mathrm{II})$ & \multirow{2}{*}{1.25} & $0.01 \mathrm{M} \mathrm{AN}$ & 1.39 & 1.24 & 90.34 \\
$\mathrm{Ni}(\mathrm{II})$ & & $1 \mathrm{M} \mathrm{NA}+0.1 \mathrm{M} \mathrm{AN}$ & 4.04 & 3.53 & 87.05 \\
$\mathrm{~Pb}(\mathrm{II})$ & & & & \\
\end{tabular}

Table-5 Removal of $\mathrm{Pb}(\mathrm{II})$ from textile industry effluents

\begin{tabular}{|l|l|l|l|l|l|}
\hline \multirow{2}{*}{ Metal Ion } & \multicolumn{2}{|c|}{ BiIW Column } & \multicolumn{2}{c|}{ BiSbW Column } \\
\cline { 2 - 6 } & Initial Conc.(mg) & Final Conc.(mg) & \% Removal & Final Conc.(mg) & \% Removal \\
\hline $\mathrm{Pb}(\mathrm{II})$ & 2.56 & 0.61 & 76.17 & 0.76 & 70.31 \\
\hline
\end{tabular}

Table-6 Efficiency of synthesized materials as dye adsorbents

\begin{tabular}{cccc}
\hline Dye solution & $\mathrm{pH}($ initial $)$ & \% of removal on BilW & \% of removal on BiSbI \\
\hline MB & 6.0 & 98.76 & 95.60 \\
MO & 7.0 & 88.82 & 82.02 \\
\hline
\end{tabular}
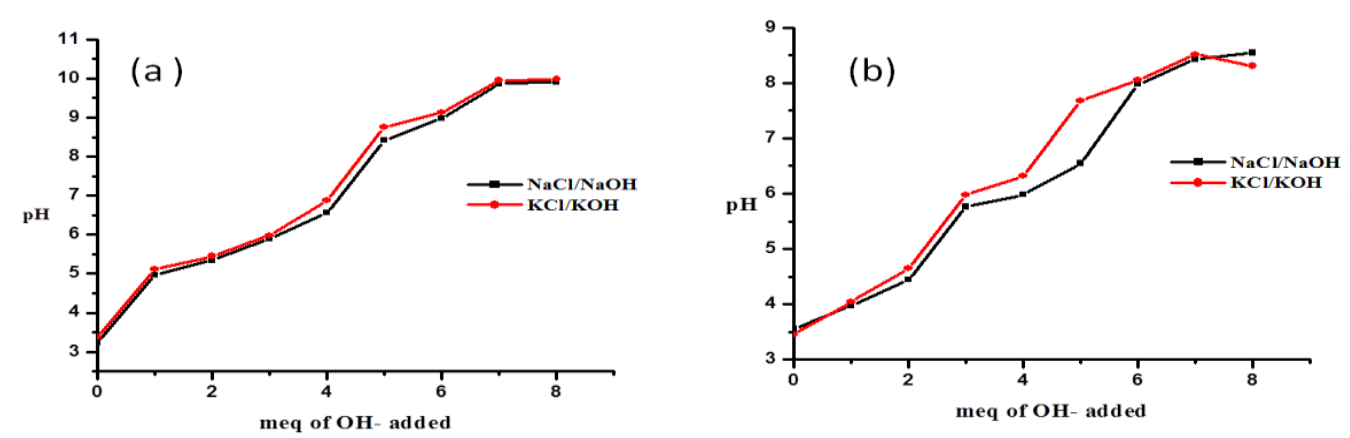

Figure-1. pH titration curves of (a ): BiSbW and (b): BiIW 


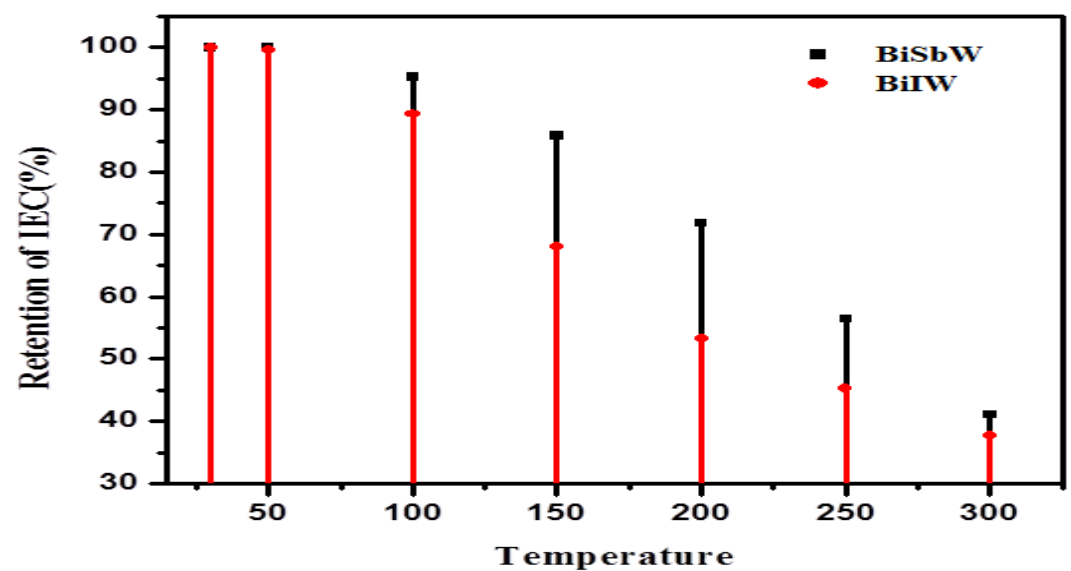

Figure-2. Effect of temperature on IEC
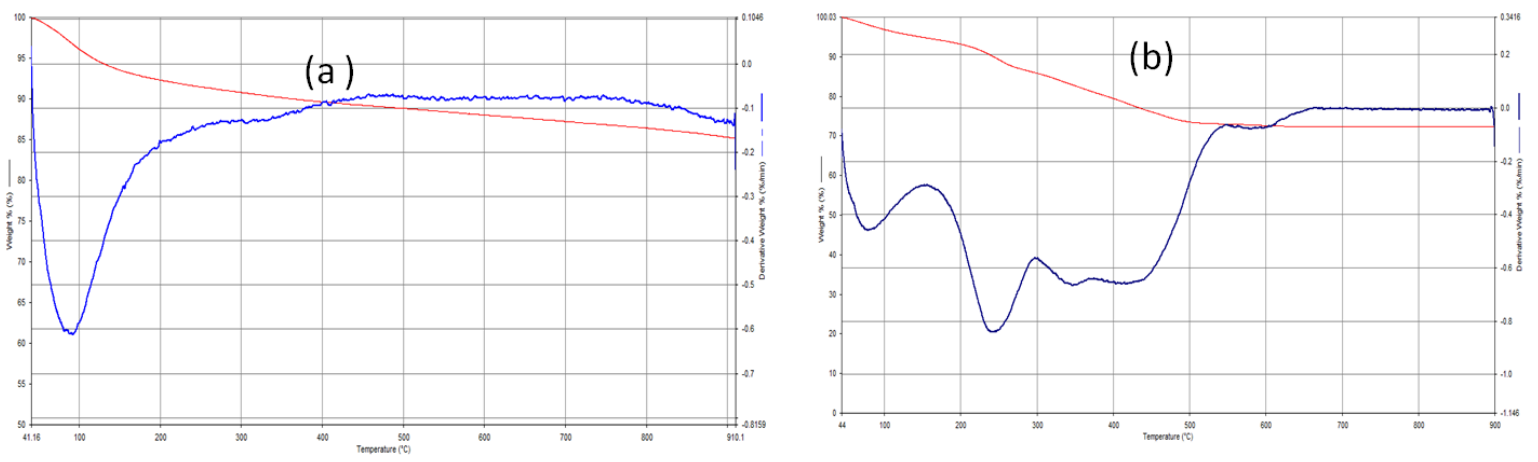

Figure-3. TG/DT analysis of the samples (a): BiSbW (b): BiIW
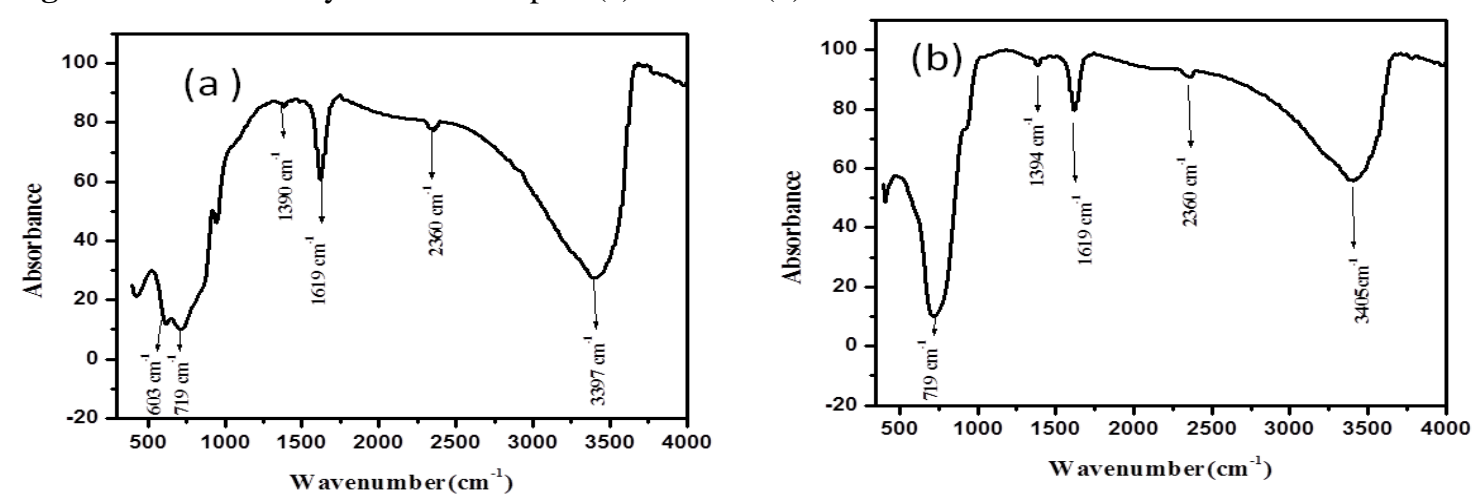

Figure-4. FTIR spectrum of (a) BiSbW and (b) BiIW

BWSb

BWI
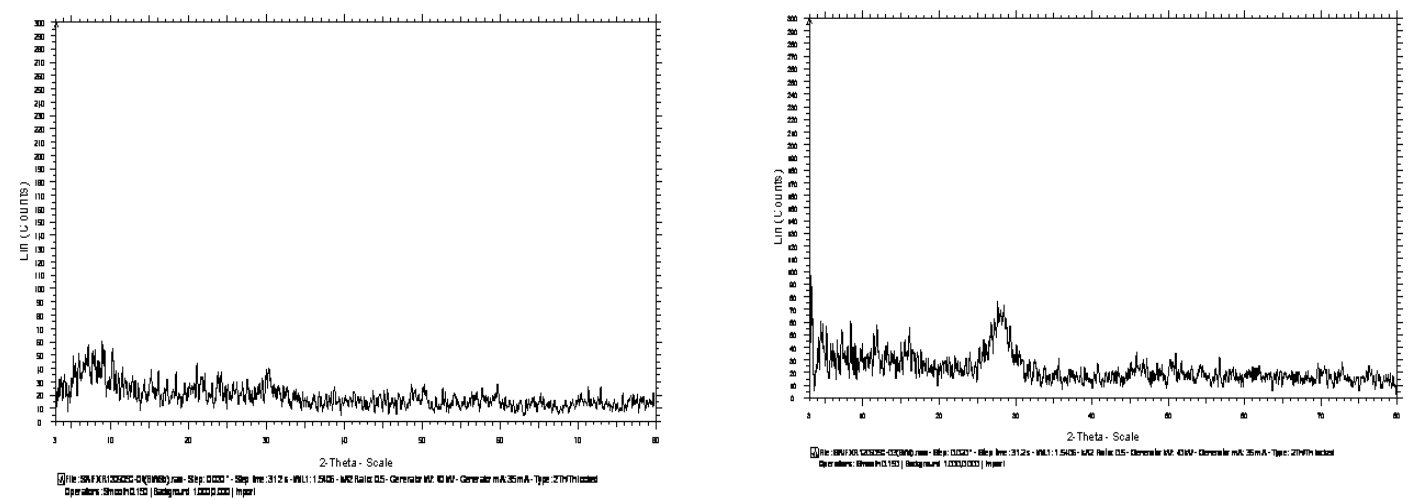

Figure-5. X- ray analysis 

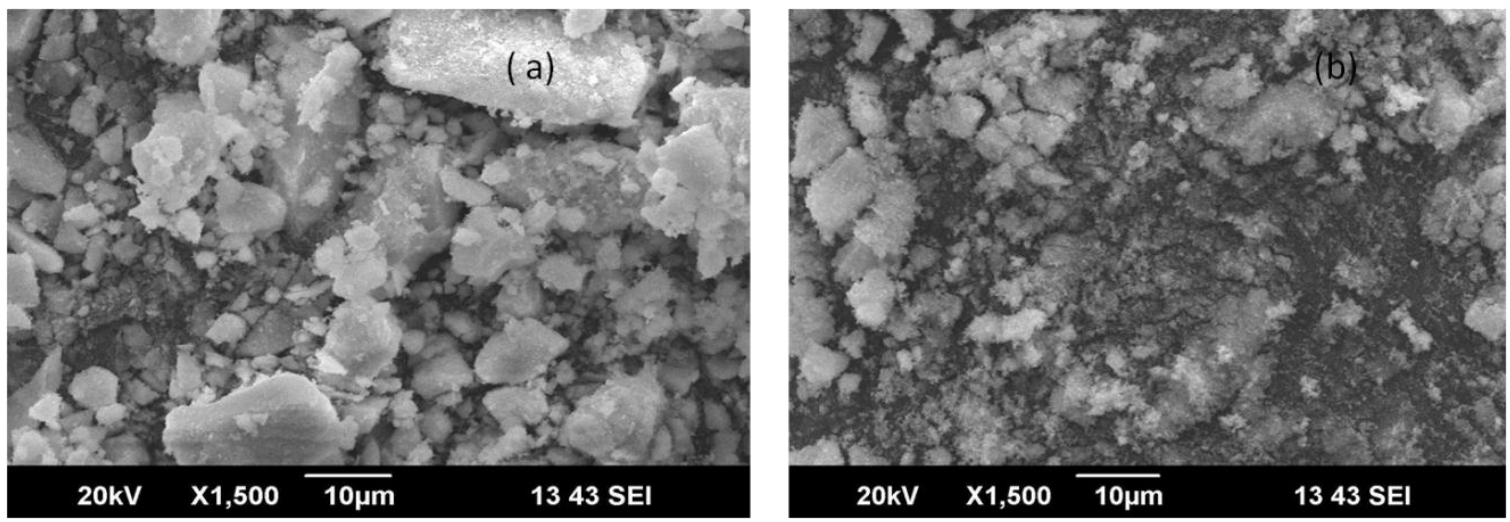

Figure-6. SEM images of (a ): BiSbW and (b): BiIW

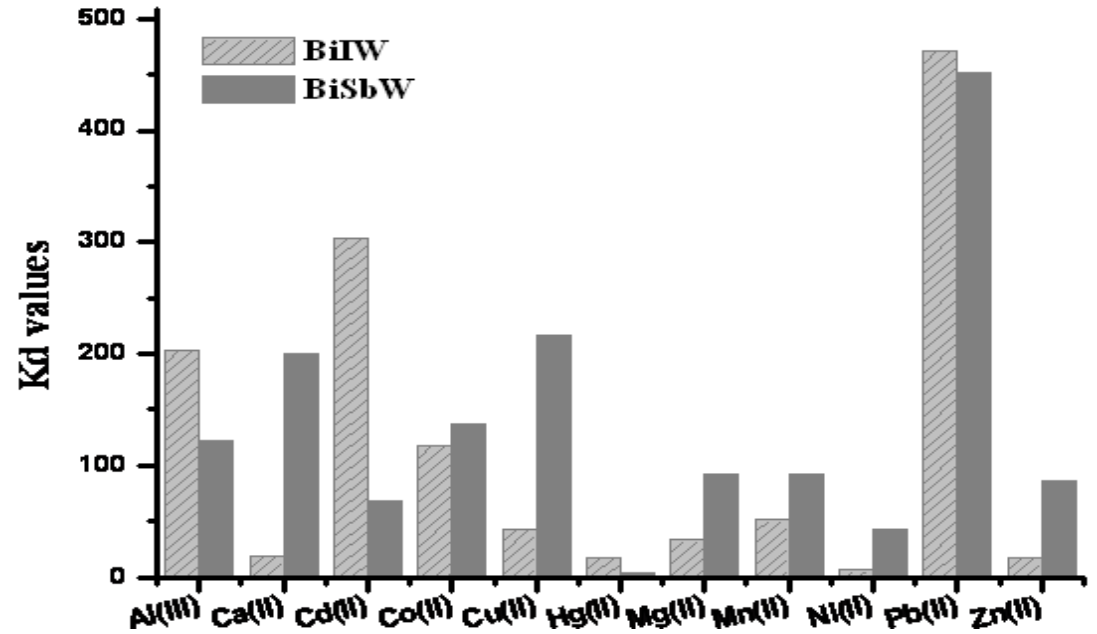

Figure-7. Distribution studies

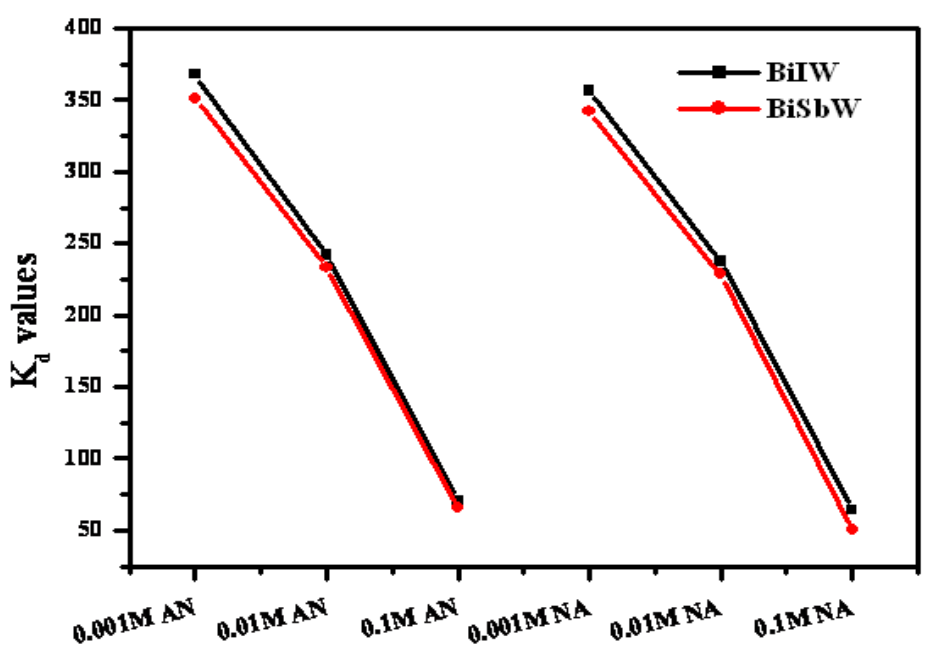

Figure-8. $\mathrm{K}_{\mathrm{d}}$ values of $\mathrm{Pb}^{2+}$ ions in different electrolyte concentration (AN: Ammonium nitrate, NA: Nitric acid) 

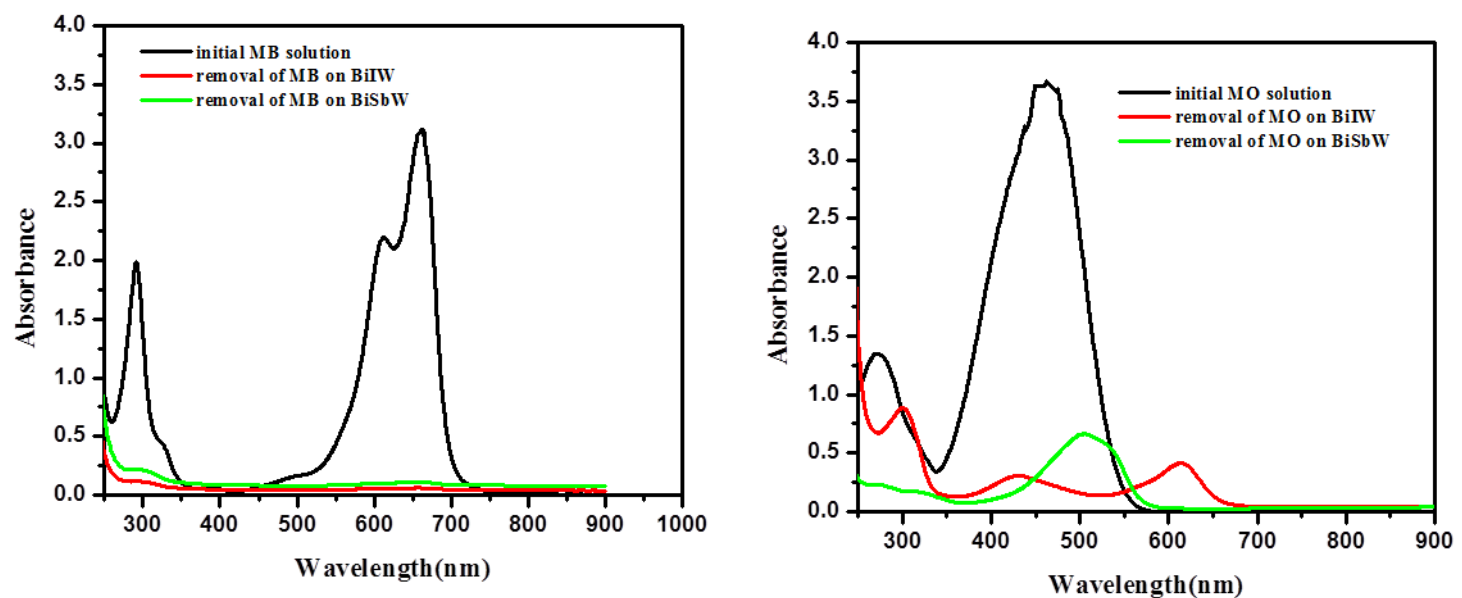

Figure-9. UV-Vis DR spectrum of dye adsorption studies.

\section{Conclution}

BiSbW and BiIW exhibit good ion exchange characteristics, thermal stability and chemical resistivity. The promising feature of these materials is their selectivity for $\mathrm{Pb}(\mathrm{II})$ ions, most toxic metal ions present in environment. Their analytical importance has been established by separating lead ions from $\mathrm{Pb}(\mathrm{II})-\mathrm{Hg}(\mathrm{II})$ and $\mathrm{Pb}$ (II)-Ni(II) binary metal ion solutions. Moreover these materials can be used as successful adsorbents for the removal of harmful organic dyes such as $\mathrm{MB}$ and $\mathrm{MO}$ from aqueous solution.

\section{Acknowledgement}

Author acknowledges the council of scientific and industrial research for awarding junior research fellowship and STIC, Cochin for providing technical facilities.

\section{References}

[1]. C. B. Amphlett, Treatment and Disposal of Radioactive Wastes (Pergamon, Oxford, 1961).

[2]. C.Janardanan and S.M.K.Nair; Preparation, properties and application of antimony (III) arsenate and antimony (III) molybdate, The Analyst, 115, 85-87, 1990

[3]. Mu. Naushad, Inorganic and Composite Ion Exchange Materials and their Applications, Ion Exchange Letters, 2, 1-14, 2009.

[4]. A.P. Gupta, G.L. Verma and Saiqa Ikram, Studies on a new heteropolyacid-based inorganic ion exchanger; zirconium(IV) selenomolybdate, Reactive \& Functional Polymers, 43, 33-41, 2000.

[5]. A. Nilchi , A. Khanchi and M. Ghanadi Maragheh, The importance of cerium substituted phosphates as cation exchanger-some unique properties and related application potentials, Talanta, 56, 383-393, 2002.

[6]. J.P. Rawat and K.P.S. Muktawat, Studies on bismuth tungstate and its use as a lead selective inorganic ion-exchanger, Chromatographia 11, 513, 1978.

[7]. P.S. Anand and D.R. Baxi, Ind. J. Tech. 16, 198, 1978.

[8]. M.K. Rahman, A.M.S. Haq and B.A. Maroof; Bismuth tellurate, an inorganic ion exchanger, J.Chromatogr. 67(2), 389, 1972.

[9]. V. Ghoulipour and S.W. Husain; Ion exchanger bismuth silicate as a novel sorbent in thin layer chromatography, Analytical Sciences, 16, 1079-1081, 2000.

[10]. Subhash Chand, Seema, Arti and Chandra Vijay Chahal, synthesis, characterization and ion exchange properties of a new ion exchange material: bismuth (iii) iodophosphate, Recent Research in Science and Technology, 3(6): 01-08,2011.

[11]. Subhash Chand, Seema, Brajesh Pal and Vijayata, Synthesis and Characterization of Bismuth (III) Tungustosilicate A New Inorganic Cation Exchanger, IOSR-JESTFT, 4(5), 30-36, 2013.

[12]. Arti and Seema, Synthesis \& Characterization of Bismuth(III) tungstomolybdate as a new cation exchanger, and its analytical applications, Elixir Appl. Chem. 53, 11858-11864,2012.

[13]. O. Samuelson, Ion exchangers in analytical chemistry (Wiley, 1952) $45 \& 117$.

[14]. N. E.Topp and K. W.Pepper, Properties of Ionexchange Resins in Relation to their Structure. Part. I. Titration Curves, J. Chem. Soc., 3299- 33031949.

[15]. M. Qureshi, J.P. Gupta and Sharma V, Synthesis of a Reproducible and Chemically Stable Tantalum antimonite. Anal Chem 45, 1901-1906, 1973.

[16]. J.P. Rawat and S.Q. Mujtaba, Synthesis and Ion Exchange Properties of Tantalum arsenate, Can J Chem 53(17): 2586-2590, 1975.

[17]. S.A. Nabi, S. Usmani, N. Rahman, Ann. Chin. France 21, 521, 1996.

[18]. S.Z. Qureshi and N. Rahman, Bull. Soc. Chim; France, 959, 1987.

[19]. M. Davis, Infrared Spectroscopy and Molecular Structure (Elsevier publishing Co, Amsterdam, 1963).

[20]. C.N.R. Rao, Chemical application of infrared spectroscopy (New York: Academic Press, 1963).

[21]. G. Socrates, Infrared Characteristic Group Frequencies (Wiley, New York, 1980).

[22]. W.A. Miller and C.H. Wilkins, Infrared Spectra and Characteristic Frequencies of Inorganic Ions, Anal. Chem. 24 1253-1294, 1952.

[23]. W. Weltner, Jr, D. McLeod, Jr, Spectroscopy of titanium, Zirconium and hafnium oxides in neon and argon matrices at $4^{\circ}$ and $20^{\circ}$ KJ. Phys. Chem. 69, 3488, 1965. 